\title{
Edge Labelling by Fusion of Intensity and Range Data
}

\author{
$G$ Zhang and $A M$ Wallace \\ Department of Computer Science, Heriot-Watt University \\ 79 Grassmarket, Edinburgh EH1 2HJ
}

\begin{abstract}
A method for labelling edge data in registered intensity and range images is presented. First, depth, orientation and intensity discontinuities are identified by one-dimensional filters aligned with the image axes. Second, the filtered outputs are combined by a maximum a-posteriori probability estimation to produce a set of edge labels at each site within the dual lattice.
\end{abstract}

\section{Introduction}

Recently, there has been interest in improving the robustness of edge detection, and achieving semantic labelling of physical edges through a combination of intensity and actively acquired range data, e.g. [1]. In this paper, we discuss a general edge model based on changes in depth, orientation and reflectance using a simple shading paradigm and sensor data corrupted by Gaussian noise. 7 categories of edge site may be distinguished within a dual lattice, i.e. edges are considered to exist between labelled pixels in the intensity and range images. These are \{blade, fold, extremal, mark, shadow, specular, no_edge\}. In object models, it is common to include only the first two categories, although invariant mark edges may also be modelled and provide a powerful cue for recognition. Blade and extremal edges are similar in terms of a local shading/depth discontinuity model, but may be distinguished by analysis of the surface normal adjacent to the edge on the surface nearest to the viewer. The position of shadow and specular edges is lighting dependent, and may be discriminated by examination of multiple intensity images.

In the simplified local edge model of Figure $1, h$ represents a change in depth, $\vec{n}_{1}$ and $\vec{n}_{2}$ are the unit surface normals of the adjacent surfaces, and $k_{d 1}$ and $k_{d 2}$ are the local diffuse reflection coefficients.

$$
I=I_{a} k_{a}+I_{p} k_{d} \frac{\vec{n} \cdot \vec{L}}{R+k}
$$

We assume a simple model for surface shading in which the reflected intensity, $I$, is the summation of light reflected from a point source, $I_{p}$, with a unit direction vector $\vec{L}$, and ambient light $I_{a}$, with respective ambient light reflection coefficients, $k_{a 1}$ and $k_{a 2}$. The

\footnotetext{
Acknowledgement: The LAIRD project is funded by the SERC/ED (GR/F38327:1551) and is a collaboration between British Aerospace, Yard Ld, the National Engineering Laboratory, and the Universities of Heriot-Watt, Edinburgh and Surrey.
} 
denominator in the second term accounts for fall-off in light intensity due to distance between surface and viewer, $R$, and includes a further constant, $k$.

Expressing $\vec{n}=(-p,-q, 1)$ and $\vec{L}=\left(-p_{L},-q_{L}, 1\right)$, changes in depth, surface normal, illumination and reflection coefficient produce changes in observed intensity. All four changes may be present at a given edge site; however, we assume a single variable changes only, summarised in Table 1 . From a single intensity and depth image, and neglecting local normal analysis in the range data, a 4 category classification of edge label is achieved, $l_{e} \in E=\{$ blade/extremal, fold, mark/shadow, no_edge $\}$.

\section{Edge classification at single sites}

We consider analysis of a vertical site though analysis at a horizontal site is similar. A row of corresponding raw range and intensity data are defined on the two sides of a vertical edge site $e_{i, j}$ by a composite observation vector $\mathbf{v}_{2 n \times 1}=\left[I_{1, j}, \ldots, I_{i, j}, \ldots, I_{n, j}, R_{1, j}, \ldots, R_{i, j}, \ldots, R_{n, j}\right]^{T}$, where $i=n / 2$ and $n$ is an even integer. A discontinuity vector is defined as $\Delta_{3 \times 1}=[\Delta I, \Delta R, \Delta N]^{T}$ where $\Delta I, \Delta R$ and $\Delta N$ are the changes of intensity, range and surface orientation obtained by filtering the raw image data $\mathbf{v}$.

$$
\Delta=\mathbf{A v} \text { and } \mathbf{A}=\left[\begin{array}{cccccccc}
a_{1} & a_{2} & \cdots & a_{n} & 0 & 0 & \cdots & 0 \\
0 & 0 & \cdots & 0 & b_{1} & b_{2} & \cdots & b_{n} \\
0 & 0 & \cdots & 0 & c_{1} & c_{2} & \cdots & c_{n}
\end{array}\right]
$$

where $\mathbf{A}_{3 \times 2 n}$ is the filter matrix. Changes in observed range, orientation and intensity are related by the geometric and shading model. We assume that the observed raw intensity and range data are conditionally independent given only the geometry of surface in view [1].

Assuming a Gaussian noise model for the intensity and range data, with standard deviation $\sigma_{I}$ and $\sigma_{R}$ respectively, then the covariance matrix $\Sigma_{2 n \times 2 n}$ of vector $\mathbf{v}$ is a diagonal matrix. Then probability density function (p.d.f.) of observation $\Delta$ is multivariate Gaussian given the no_edge label, where the covariance matrix $\tilde{\Sigma}$ of vector $\Delta$ is $\tilde{\Sigma}=\mathbf{A} \Sigma \mathbf{A}^{T}$. Choosing filter coefficients in $\mathbf{A}$ such that $a_{k}=-a_{n+1-k}$, $b_{k}=-b_{n+1-k}$ and $c_{k}=c_{n+1-k}$, the covariance matrix $\tilde{\Sigma}$ is simplified to the diagonal matrix of equation (3).

$$
\tilde{\Sigma}=\left[\begin{array}{ccc}
\left(\sum_{1}^{n} a_{i}{ }^{2}\right) \sigma_{I}^{2} & 0 & 0 \\
0 & \underset{1}{\left(\sum_{i}^{n} b_{i}{ }^{2}\right) \sigma_{R}^{2}} & \underset{1}{\left(\sum_{i}^{n} b_{i} c_{i}\right) \sigma_{R}^{2}} \\
0 & \underset{1}{\left(\sum_{i}^{n} b_{i} c_{i}\right) \sigma_{R}^{2}} & \underset{1}{\left(\sum_{1}^{n} c_{i}{ }^{2}\right) \sigma_{R}^{2}}
\end{array}\right]=\left[\begin{array}{ccc}
\left(\sum_{1}^{n} a_{i}{ }^{2}\right) \sigma_{I}^{2} & 0 & 0 \\
0 & \underset{1}{\left(\sum_{i}^{n} b_{i}{ }^{2}\right) \sigma_{R}^{2}} & 0 \\
0 & 0 & \left(\sum_{1}^{n} c_{i}{ }^{2}\right) \sigma_{R}^{2}
\end{array}\right]
$$


Therefore, the observed filtered changes in range, orientation and intensity are conditionally independent given the no_edge label. The p.d.f. $p\left(\Delta \mid l_{e}=n o \_e d g e\right)$ is simplified into the multiplication of three one-dimensional p.d.f.'s. In general, $\Delta R$ and $\Delta N$ are independent given the edge label $l_{e}$. From Bayes theorem, the a-posteriori probability of an edge label $l_{e}$, given an observation vector $\Delta$, can be computed by the equation for $l_{e} \in E$.

$$
P\left(l_{e} \mid \Delta\right)=\frac{P\left(l_{e}\right) p\left(\Delta \mid l_{e}\right)}{P(\Delta)}=z \frac{P\left(l_{e} \mid \Delta I\right) P\left(l_{e} \mid \Delta R\right) P\left(l_{e} \mid \Delta N\right)}{\left(P\left(l_{e}\right)\right)^{2}}
$$

where $z=p(\Delta I) p(\Delta R) p(\Delta N) / p(\Delta)$ is a constant once an observation is obtained. The prior probabilities $P\left(l_{e}\right)$ are constant and are estimated from known image properties. To evaluate the numerator, we adopt the method in [2],

$$
P\left(l_{e}=n o_{-} e d g e \mid \Delta\right)=\exp \left(-\frac{\Delta^{2}}{\sigma^{2}}\right) \text { and } P\left(l_{e}=\text { edge } \mid \Delta\right)=1-\exp \left(-\frac{\Delta^{2}}{\sigma^{2}}\right)
$$

where $\Delta$ may be either $\Delta I, \Delta R$ or $\Delta N$, and $\sigma^{2}$ the corresponding variance in equation (3). From equation (4) the estimate of the edge label at a single site is that for which $P\left(l_{e} \mid \Delta\right)$ is a maximum.

\section{Results and Discussion}

Figure 2 illustrates the application of the method to a synthetic image. The original test data is illustrated in the top row. Succeeding rows show the labelled mark, blade and fold edges with noise levels $\sigma_{I}=\sigma_{R}=1$ and 2 respectively. The filter coefficients of matrix $\mathrm{A}$ are calculated from the first $\left(a_{i}, b_{i}\right)$ and second $\left(c_{i}\right)$ derivatives of a Gaussian function of standard deviation 1 pixel, truncated at $n=10$. The prior probabilities $P\left(l_{e}\right)$ of blade, fold, mark and no_edge labels are estimated as $0.08,0.07$ and 0.06 and 0.79 respectively. Range and depth data are scaled in the range 0-255. The majority of mark and blade edges are classified correctly, but some fold edges are missed due to the weak response of orientation changes. These initial results are promising, but indicate the necessity for further development and more systematic testing. Currently, we are considering the effects of misregistration and sparse depth data in real images, and improving the initial classification by neighbourhood analysis.

\section{References}

1. Gamble, E. B., Geiger, D., Poggio, T., and Weinshall, D, "Integration of Vision Modules and Labeling of Surface Discontinuities," IEEE Trans. Syst., Man, Cybern., Vol. 19, (6), pp. 1576 - 1581, (1989).

2. Hancock, E. R. and Kittler, J., "Edge-Labeling Using Dictionary-Based Relaxation," IEEE Trans. Pattern Anal. Machine Intell., Vol. 12, (2), pp. 165 181, (1990). 


\begin{tabular}{|c|c|c|r|}
\hline \multirow{2}{*}{$\begin{array}{c}\text { Edge } \\
\text { Labels }\end{array}$} & \multicolumn{3}{|c|}{ Changes of Range, Normal and Intensity } \\
\cline { 2 - 4 } & $\Delta R$ & $\Delta N$ & $\Delta I$ \\
\hline blade & $h$ & - & $I_{p} k_{d 1}\left(p_{L} p_{1}+q_{L} q_{1}+1\right) h /\left(\left(R_{1}+k\right)\left(R_{1}+k+h\right)\right)$ \\
\hline fold & $\varnothing$ & $\begin{array}{c}\left(p_{1}-p_{2},\right. \\
\left.q_{1}-q_{2}, 0\right)\end{array}$ & $I_{p} k_{d 1}\left(\left(p_{1}-p_{2}\right) p_{L}+\left(q_{1}-q_{2}\right) q_{L}\right) /\left(R_{1}+k\right)$ \\
\hline mark & $\varnothing$ & $\varnothing$ & $I_{a}\left(k_{a 1}-k_{a 2}\right)+I_{p}\left(k_{d 1}-k_{d 2}\right)\left(p_{L} p_{1}+q_{L} q_{1}+1\right) /\left(R_{1}+k\right)$ \\
\hline shadow & $\varnothing$ & $\varnothing$ & $I_{p} k_{d 1}\left(p_{L} p_{1}+q_{L} q_{1}+1\right) /\left(R_{1}+k\right)$ \\
\hline no_edge & $\varnothing$ & $\varnothing$ & $\varnothing$ \\
\hline
\end{tabular}

Table 1: Correspondence between edge label and discontinuities

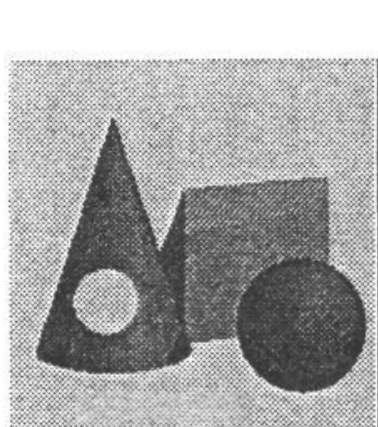

(a)

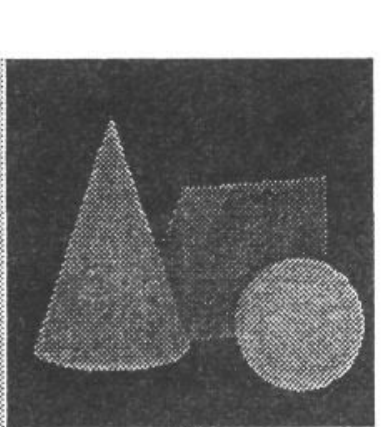

(b)

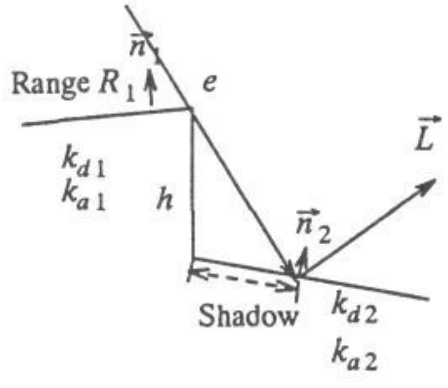

Figure 1: Edge shading model

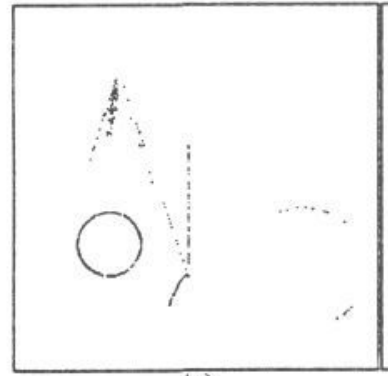

(c)

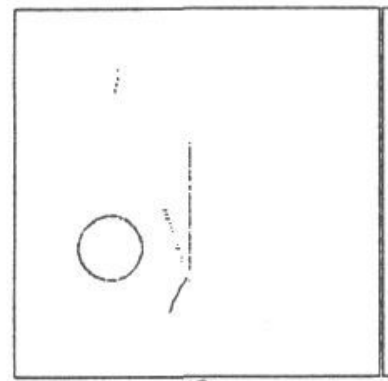

(f)

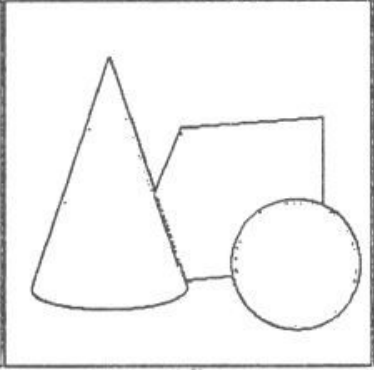

(d)

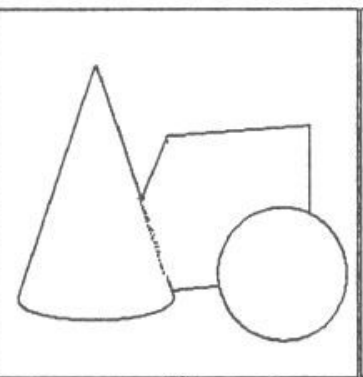

(g) (e)

(h)

Figure 2: Initial classification results 
